\title{
BMJ Open Cocreation of a digital patient health hub to enhance education and person- centred integrated care post hip fracture: a mixed-methods study protocol
}

\author{
Lalit Yadav (D) , ,2 Tiffany K Gill (1) , ${ }^{3}$ Anita Taylor, $^{2}$ Unyime Jasper, ${ }^{1}$ Jen De Young, ${ }^{2}$ \\ Renuka Visvanathan, ${ }^{1,4}$ Mellick J Chehade (D) ${ }^{1,2}$
}

To cite: Yadav L, Gill TK, Taylor A, et al. Cocreation of a digital patient health hub to enhance education and personcentred integrated care post hip fracture: a mixed-methods study protocol. BMJ Open 2019:9:e033128. doi:10.1136/ bmjopen-2019-033128

- Prepublication history and additional material for this paper are available online. To view these files, please visit the journal online (http://dx.doi org/10.1136/bmjopen-2019033128).

Received 22 July 2019 Revised 04 November 2019 Accepted 21 November 2019

Check for updates

(C) Author(s) (or their employer(s)) 2019. Re-use permitted under CC BY-NC. No commercial re-use. See rights and permissions. Published by BMJ.

For numbered affiliations see end of article.

Correspondence to

Lalit Yadav;

lalit.yadav@adelaide.edu.au

\section{ABSTRACT}

Introduction Older people with hip fractures often require long-term care and a crucial aspect is the provision of quality health information to patients and their carers to support continuity of care. If patients are well informed about their health condition and caring needs, particularly posthospital discharge into the community setting, this may support recovery and improve quality of life. As internet and mobile access reach every household, it is possible to deliver a new model of service using a digital education platform as a personal health hub where both patients and their providers of care can establish a more efficient information integration and exchange process. This protocol details proposed research, which aims to develop a 'model of care' by using a digital health solution that will allow delivery of high quality and patient-centred information, integrated into the existing process delivered within the community setting.

Methods and analysis This phase of the study uses a pragmatic mixed-methods design and a participatory approach through engagement of patients, their carers and healthcare providers from multiple disciplines to inform the development of a digital health platform. Quantitative methods will explore health literacy and e-health literacy among older people with hip fractures admitted to the two public tertiary care hospitals in Adelaide, South Australia. Qualitative methods will provide an understanding of aspects of content and context required for the digital health platform to be developed in order to deliver quality health information. The study will use appropriate theoretical frameworks and constructs to guide the design, analysis and overall conduct of the research study. The scope of the study intends to ultimately empower patients and their carers to improve self-management and to better use coordinated services at the community level. This could prevent further falls including associated injuries or new fractures; reduce new hospital admissions and improve confidence and engagement by limiting the psychologically restrictive 'fear of falls'.

Ethics and dissemination The study has been approved by the Human Research Ethics Committee of the Central Adelaide Local Health Network, South Australia (SA) Health, Government of South Australia and the University of Adelaide Human Research Ethics Committee. Findings from the study will be published in suitable peer-reviewed journals and disseminated through workshops or conferences.
Strengths and limitations of this study

- The proposed study will use a mixed-methods approach, which would provide a unique perspective around patient educational and information needs during the hip fracture care pathway, through a combination of the distinct strengths of each methodology.

- The study intends to provide enriched data through interpretation of results, using different theoretical frameworks, including the best practice clinical guidelines, contemporary models of implementation science and behavioural change to guide the design and analysis of study findings.

- Involvement of patients and carers is a key feature in the design of this study.

- A quantitative survey considering only two hospital settings could be a limitation of the study as the study findings may not be generalisable to the wider Australian context or internationally.

\section{INTRODUCTION}

Management of hip fracture in older adults poses significant challenges to delivering quality healthcare due to multiple medical, social and isolation issues, including frailty. ${ }^{1}{ }^{2}$ Worldwide, hip fractures are projected to increase from 1.7 million in 1990 to 6.3 million in 2050 due to significant increases in ageing and life expectancy. ${ }^{3-5}$ In 2000, an estimated 9 million osteoporotic fractures occurred worldwide and the annual costs for treatment have been assessed to be around US $\$ 20$ billion in the USA and $€ 30$ billion in the European Union, ${ }^{6}$ with $72 \%$ of this cost incurred for the management of hip fractures. Following a hip fracture, the use of health services extends beyond the initial hospitalisation for at least 1 year, with much of the healthcare costs attributable to subsequent long-term care. ${ }^{7-10}$ Such patients are at high risk of complications with devastating outcomes, loss of independence, 
decreased mobility and reduced quality of life. ${ }^{11}$ Postdischarge, most of these patients attend orthopaedic outpatient departments, which are located in hospitals where access can be difficult, as patients rely on family or ambulance services to attend. For falls prevention, they need to access services generally located in the community, and general practitioners for management of existing comorbidities. This often leads to disconnected pathways of care contributing to discontinuation of appropriate care due to lack of integration between different services. The difficulty related to continuing care could also be due to low empowerment among older people with hip fractures or consumers of health services, in general. ${ }^{12}$ Patients and their carers may lack the skills to understand complex instructions related to medication, self-monitoring and self-management, follow-up schedules and prevention behaviours. Adequate health literacy skills are important for understanding surgical procedures, informed consent and adhering to postsurgical instructions. Health literacy is a patient factor that can be influenced by both patient skill level, as well as by the information, communication and education provided to them. ${ }^{13}$ Therefore, a single integrated care plan management system is needed that empowers the patient and their carers for both home and community management. ${ }^{14} 15$ To be successful, the plan must adopt a systematic approach to ensure that individuals with one or more long-term conditions, and their health and care providers, have more productive and equal conversations, focused on what matters most to the individual. ${ }^{16}$ With the advancement of modern information technology, it should be possible to seamlessly integrate different services for older people with hip fracture from acute hospital (tertiary) care to community rehabilitation and management through provision of quality health information. There is an imperative to reorient services to the community so that they can be delivered closer to the patients and in partnership with the consumers and primary/aged care services.

The proposed research aims to develop a "model of care' by using a digital health solution that will allow delivery of high-quality and patient-centred information, integrated into the existing process, delivered within the community setting. The research will be conducted in different phases, incorporating a cocreation approach involving patients and their carers, primary/aged care clinicians, physiotherapists, dieticians and hospital-based clinicians through an iterative process. This first phase of the study would inform the development of a prototype, a digital health platform (phase 2). This will be further pilot tested for usability in the next stage (phase 3). Thus, this study protocol paper exclusively deals with the detailed methods for the first phase (figure 1). The objectives of this phase of the study are first, to understand the e-literacy level of patients with hip fractures in terms of their current use of technology in accessing health information and their likelihood of using such systems through their computers or mobile and other digital applications (context). Second, to explore specific health information requirements (content) for people with hip fractures, particularly after their discharge from the acute hospital

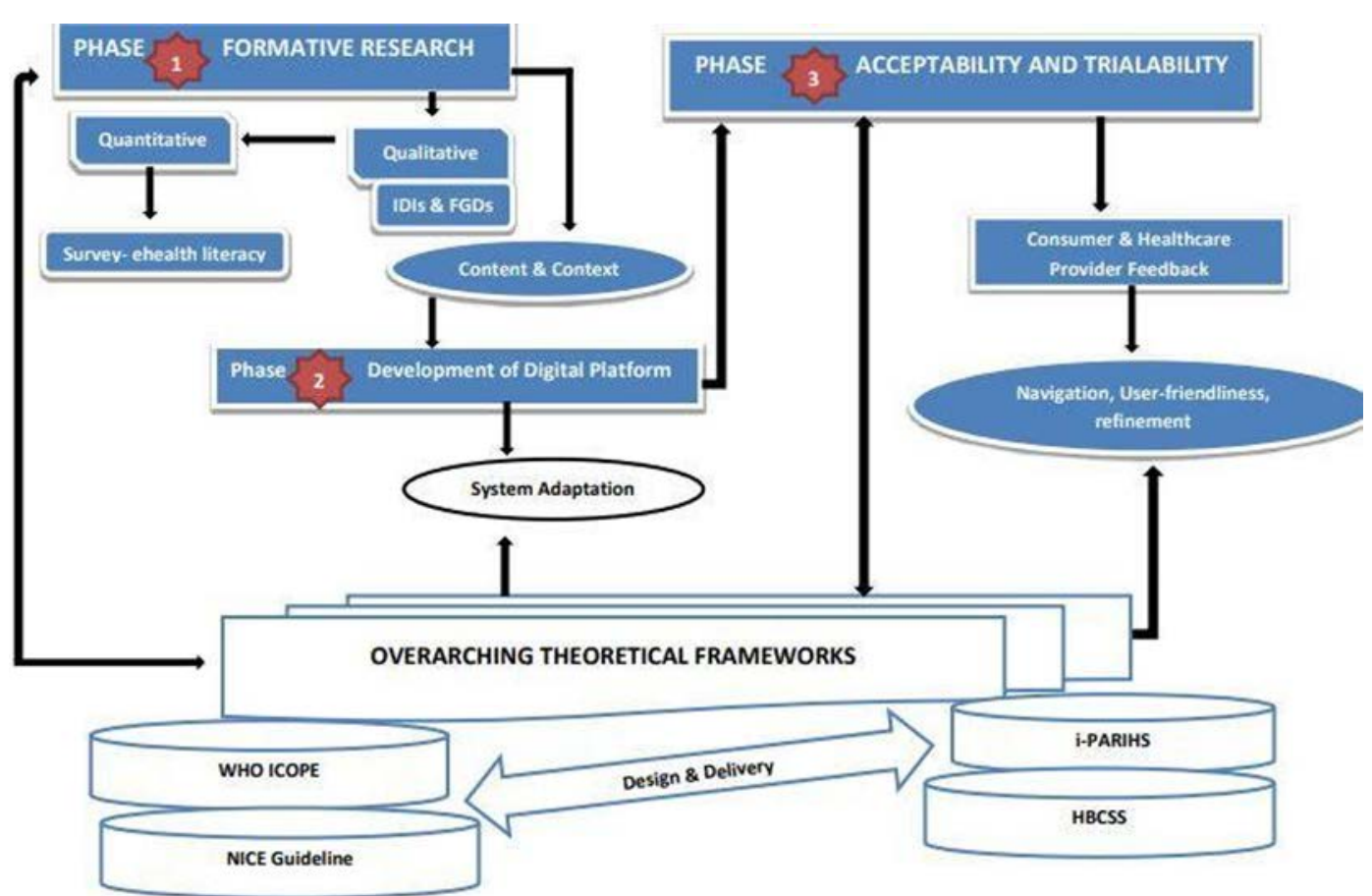

Figure 1 Conceptual Framework-Digital Health Patient Education Platform. FGD, focus group discussion; HBCSS, Health Behaviour Change Support Systems; IDI, in-depth interview; i-PARIHS, integrated-Promoting Action on Research Implementation in Health Services; NICE, National Institute for Health and Care Excellence; WHO-ICOPE, WHO integrated care for older people. 
setting, from the perspective of patients and their carers; clinicians and residential care providers. Third, to determine important factors that need to be considered at the time of designing digital health educational platform for the patients with hip fractures (system) including potential barriers and facilitators around future use of such technologies.

\section{METHODS}

\section{Study design}

The proposed study will be using pragmatic design including mixed-methods research and a participatory approach through engagement of patients, their carers and healthcare providers. ${ }^{17}$ Previous research clearly states that the ultimate success of health-related technologies depends on whether the intended users (eg, patients) find the developed applications useful. ${ }^{18}$ The process of cocreation allows end users to directly influence how the technologies take shape in order to increase ultimate usability. Evidence indicates that involving end users throughout the technology development process, substantially reduces development time and allows easy translation of technologies to practice, as usability problems are identified and resolved before the systems are launched. ${ }^{19} 20$ The study will be conducted at two hospital sites in Adelaide; the Royal Adelaide Hospital (RAH) and the Queen Elizabeth Hospital (TQEH).

\section{Theoretical framework}

This study will be using theoretical frameworks to guide the process of design, development and conduct of the study in real-world setting. These are the National Institute for Health and Care Excellence (NICE) guideline on hip fracture management; WHO's guideline on community-level interventions to manage declines in intrinsic capacity through an integrated care approach for older people (WHO-ICOPE); Health Behaviour Change Support Systems (HBCSS) and integrated- Promoting Action on Research Implementation in Health Services (i-PARIHS). ${ }^{21-24}$

According to the recently available NICE guideline on hip fracture management, good quality advice, reassurance, information and education were highlighted by patients as important factors in the recovery process. ${ }^{22}$ Examining older people from the perspective of their intrinsic capacity and the outside environment in which they live helps to understand why health services should be oriented towards the most relevant outcomes that affect older people on a day-to-day basis. Further, this approach could eliminate unnecessary treatments, reduce polypharmacy and associated side effects and hopefully improve the overall quality of life for older people. The WHO-ICOPE guideline recommends evidence-based interventions to manage common declines in capacity among older people. These conditions were recommended because they express reductions in physical and mental capacities, as outlined in a WHO framework on healthy ageing and are strong independent predictors of mortality and care dependency in older age. ${ }^{21}$ The WHOICOPE framework will guide this proposed research study in terms of developing more comprehensive and holistic educational content for the posthospital discharge setting and not just restricted to the specifics of a hip fracture injury.

The study further intends to use concepts from a contemporary theoretical framework around computer mediated communication and persuasive roles. This research domain is termed as HBCSS. ${ }^{23}$ HBCSS has been defined as a sociotechnical information system that forms, alters or reinforces attitudes, behaviours or acts of complying, without using deception or coercion. ${ }^{23} 25$ The three intertwined components of HBCSS are content, system and context. Content within HBCSS is often referred to as text or video; system is the technological mode and features used to deliver the content; and context is related to the specific organisational context or setting in which the proposed technological solution is desired to be implemented. ${ }^{23}$ Due to the complex nature of HBCSS and as it is still evolving as a research discipline, there is a need to combine established theoretical frameworks such as i-PARIHS to further understand the implementation context and guide the design and development of the proposed research study. ${ }^{246}$

According to i-PARIHS, successful implementation involves facilitation of innovation, recipients and context, taking account of them together and how they interrelate with each other. The construct of 'innovation' includes explicit knowledge available through evidence and tacit, practice-based knowledge, which is considered to be influencing when it comes to implementation. The construct of 'recipient' includes those people who are affected by and influence implementation process and outcomes at both individual and collective team levels. 'Context' exists as different layers at micro, meso and macro, and is further defined in terms of resources, culture and leadership. It goes beyond local context to wider organisational, health system or external policy influences. ${ }^{24}$

While there are some overlapping constructs or concepts with certain limitations in each of the frameworks, a combination approach to guide different stages of the research process and exploring these constructs or concepts from different perspectives will provide robust understanding around study results.

\section{Quantitative method}

\section{Inclusion and exclusion criteria}

Consecutive patients aged 65 years and above with a hip fracture injury admitted to either of the two public tertiary care centres in South Australia (RAH and TQEH), and who could carry out their activities of daily living, independently prior to hospital admission, will be invited to participate in the study. Activities of daily living will be extracted from the case records as this is examined by an orthogeriatric nurse as part of the existing practice. Those patients giving written informed consent will be 
recruited in the study irrespective to their levels of (e) health literacy skills. Those patients highly dependent on medical care who may be unable to give consent, according to the treating clinician's discretion, will be excluded from the study.

\section{Sample size and questionnaire}

Approximately, 100 participants will be recruited from the two hospital sites over a period of 6 months. This sample size is based on realistic consideration around recruitment of participants in the given setting and time frame, and can be presented as a representative snapshot of the people admitted with hip fracture in these two hospitals. A published study on health literacy among elderly patients with a heart failure was also considered to justify our sample size, as a surrogate. ${ }^{27}$ A structured survey questionnaire has been developed primarily to assess health literacy and e-health literacy using a validated 14-item health literacy scale ${ }^{28}$ and electronic health literacy scale. ${ }^{29}$ Frailty status of the participants will be assessed through a validated Modified Fried Frailty Phenotype. According to this phenotype, frailty is present when three or more of the following criteria are met: unintentional weight loss, weak grip strength, self-reported exhaustion, slowness and low physical activity level. On the other hand, when one or two of these criteria are met, respondents are classified as prefrail. However, for the purpose of this study, we will be using a dichotomous Frailty Phenotype; non-frail (0-2 deficits, combining non-frail and prefrail categories) and frail (3+deficits). ${ }^{30}$ The last section of the questionnaire consists of information around hospital hip fracture care and management. The variables in the dataset have been recommended as part of the Australia and New Zealand Hip Fracture Registry and the Global Fragility Fracture Network. ${ }^{11}$ The required information can be extracted from the patient hospital records and admission data. Approximately 20-30 min will be required to complete the survey questionnaire (see online supplementary appendix 1 ).

\section{Statistical analysis plan}

The quantitative data will be analysed to address the first objective of the study related to general health literacy and e-health literacy among older people with hip fracture. Apart from frequencies of basic demographic information, current use of information technology for accessing health information and the likelihood of using the future technological solutions will also be analysed. This will help determine the likely scenario of usability of potential digital health educational platform. The participants will be classified into two groups, non-frail and frail and the differences in the data between the two groups will be analysed using Student's t-test or $\mathrm{X}^{2}$ test and also separately for each sex. In addition, multivariable logistic regression analysis will be undertaken, adjusted for relevant covariates (age, sex, body mass index and education).

\section{Qualitative method}

The qualitative component of this phase of the study will consists of in-depth interviews (IDIs) and focus group discussions (FGD) conducted with healthcare providers from different disciplines, patients, their carers and aged care providers. Orthopaedic and geriatric consultants, residents and nursing staff, physiotherapist, dietician and fracture liaison nurse will be included. Approximately, 15 IDIs and 4 FGDs will be conducted. An interview schedule has been prepared separately for patients, their carers and healthcare providers (see online supplementary appendices 2-4) and separate FGD guides for healthcare providers and patients along with their carers (see online supplementary appendices 5 and 6 ). Views of patients, along with their carers, will be explored around specific health information requirements (content) for people with hip fractures, particularly after discharge from the acute hospital setting. This component will help to determine important factors that need to be considered at the time of designing a digital health educational platform including potential barriers and facilitators around future use of such technologies. Thus, addressing the second and third objectives of this phase of study.

The audio recording will be transcribed verbatim and analysed according to themes. The researcher will analyse the data simultaneously with data collection until data saturation is reached. Different themes which emerge from the data will then be compared and interpreted according to the constructs of HBCSS theoretical framework. ${ }^{20}$

\section{Data management, ethics and dissemination}

A participant information sheet will be provided to potential participant prior to recruitment. This will incorporate the study description including participation requirements, benefits, confidentiality and data protection, the written informed consent process and the opportunity to withdraw from the study at any stage in the project. Findings from the study will be published in suitable peerreviewed journals and disseminated through workshops or conferences.

\section{Confidentiality and data security}

Any information obtained in connection with this research project that can identify study participants will remain confidential. The collected information will only be used for the purpose of this research project and it will only be disclosed with participant's permission, except as required by the law. The IDIs and FGDs recording will be transferred from the audio recording device onto a secure server, soon after the data collection is completed. The data will be deleted from the recording device after ensuring all of the data have been successfully transferred to the secured server drive. The transferred recording on the server will be de-identified and only accessible to the researchers, working on this study project. 


\section{Patient and public involvement}

Older people including former patients with hip fractures, their family members and representatives from residential aged care providers were involved in the early conceptualisation phase through a consumer research showcase event. This event was hosted by Multicultural Aged Care South Australia and National Health and Medical Research Council (NHMRC) Centre for Research Excellence in Frailty and Healthy Ageing, Adelaide Medical School, the University of Adelaide. One of the authors (LY) led the discussion with the event participants to understand the direction of this research process and informed the study design. Further, the study steering group will also include representation from patients, consumers group and residential aged care providers to guide the conduct of the study at each phase and will be closely monitored.

\section{DISCUSSION}

Hip fractures in older age require multidisciplinary integrated care and are often regarded as a surrogate marker of how the health system deals with frail, older patients. ${ }^{31}$ A study relating to consumers' perspective conducted in Sweden demonstrated that following an event of hip fracture, patients not only have restricted mobility but also lose their confidence and self-efficacy due to the complex recovery process consisting of both physical and psychological strain. The study further concluded that even after 4 months postsurgery, the previously healthy and independently living felt hip fractures affected their day-to-day life. ${ }^{32}$ Another study revealed that due to exposure to the ward culture at the time of acute hospital admission, patients become passive and insecure about their future life situation. This suggest patients believe in recovery but lack psychological support to regain prefracture status ${ }^{33}$ or have inadequate empowerment. ${ }^{34}$

The aim of our proposed research is to develop a 'model of care' by using a digital health patient education platform. The development of this digital health educational platform will go through an iterative process, across the three phases. In each phase, patients, their carers including their family members and relevant healthcare providers will be engaged through a cocreation process.

Patients and their carers are interested in being involved in the decision-making process about the management of their condition. Increasingly, emphasis has been given to provide solutions, which assist patients with more information and enable them to actively participate in their care process, including management of their expectations about the recovery process prior to hospital discharge. ${ }^{35} 36$ Management of conditions like osteoporosis, which often lead to hip fractures, require complex interventions; of which patient education appears to be the most important component. ${ }^{37}$ Educating patients requires provision of good-quality health information to encourage patient participation in healthcare and ensuring that patients have greater power, protection and choice in key aspects of their care. ${ }^{38}$ Also, patient information is a key component around effective self-management. ${ }^{39}$

Patient education centres around the assumption that patients who are better informed about their condition and management will be more likely to adopt positive health behaviours ${ }^{40}$ and will therefore improve, maintain or slow deterioration of their health status. However, this view of patient education does not acknowledge the role of patient opinions and choices, and implies that health professionals set the education agenda and define optimal health behaviours. ${ }^{41}$ Attitudes, beliefs and behaviours are considered to be important factors in influencing information needs of the patients, in addition to contextual factors and the format of educational resources. ${ }^{42}$ Our study design is based on using sound theoretical frameworks including clinical guidelines. Each framework contributes in a different manner to the process; the NICE and WHO-ICOPE guidelines will help to guide the best practice around the development of information 'content' for the potential digital health solution. HBCSS will guide the development of digital health 'system' and i-PARIHS focuses on the 'process of implementation' from a health system perspective. There are some overlapping constructs between these frameworks alongside certain limitations. However, simultaneously, the study will also use existing knowledge around the best practices from local healthcare providers' perspective in South Australia.

In a recent study by Brookes, over 228000 comments posted to the National Health Service (NHS) choice website were evaluated both quantitatively and qualitatively through a computer-assisted programme. The study suggested patients' perceptions for possible areas of improvement within various aspects of NHS service provision. High priority was given to the interpersonal aspects of healthcare provider interaction as well as system or organisational issues in coordinating services. ${ }^{43}$ Similarly, by involving the user in a participatory design ensures consumers' requested functionalities can be incorporated to optimise the usability of the potential solution and simultaneously empower healthcare providers. ${ }^{44}$

A recent study demonstrates that older people with hip fractures can respond well to modern technological solutions used for health knowledge in spite of their limited use. ${ }^{45}$ Technological advancement should consolidate relevant information in a broad-reaching manner, with real-time support to patients and their carers in their journey from diagnosis to follow-up. ${ }^{35}$ Technology can potentially empower and build the capacity of primary healthcare providers to provide integrated care that channels appropriate expertise to the patient and brings specialty consultations closer to the community. Furthermore, technology helps to engage patients through improved communication and fostering selfmanagement skills for their chronic conditions. ${ }^{46}$ For success, it must adopt a systematic approach to engage people with chronic disease conditions and complex care needs, along with their care providers, and ensure they 
have an equal say in the matters about the management of their disease condition. ${ }^{16}$ With the advancement of modern information technology, it should be possible to integrate seamlessly the provision of desired educational information for older people with hip fracture from acute hospital (tertiary) care to community rehabilitation and management.

\section{Author affiliations}

${ }^{1}$ Center for Research Excellence in Frailty and Healthy Ageing, Adelaide Medical School, The University of Adelaide, Adelaide, South Australia, Australia

${ }^{2}$ Orthopaedic and Trauma, Royal Adelaide Hospital, Adelaide, South Australia, Australia

${ }^{3}$ Adelaide Medical School, Faculty of Health and Medical Sciences, The University of Adelaide, Adelaide, South Australia, Australia

${ }^{4}$ Aged and Extended Care, The Queen Elizabeth Hospital, Woodville South, South Australia, Australia

Acknowledgements The authors would like to acknowledge the consumer group who representated the research showcase event-2018, held in Adelaide, South Australia for their valuable inputs. Further, individual team members for their encouragement and support for the study, form the following units: CRE-Frailty and Healthy Ageing at the Adelaide Medical School, the University of Adelaide; Department of Orthopaedic and Trauma, Royal Adelaide Hospital; and Aged and Extended Care, The Queen Elizabeth Hospital, Adelaide, South Australia.

Contributors LY, TKG, RV, AT and MJC contributed in the study conceptualisation, following iterative process of discussion. LY in routine discussion with TKG conducted the background literature search around theoretical framework and methodology to guide the conduct of the pragmatic study approach. LY write the initial first draft under the guidance of MJC, RV and TKG. The draft was reviewed and critical inputs were provided by TKG, AT, UJ, JDY, RV and MJC. This final version of the manuscript incorporates comments and edits from the authors and approved by all.

Funding This study is financially supported through NHMRC Centre for Research Excellence in Frailty and Healthy Ageing at the Adelaide Medical School, the University of Adelaide (NHMRC Grant ID- 1102208). LY and UJ are supported through Commonwealth Government of Australia Research Training Program Scholarship and the University of Adelaide International Scholarship respectively. No external sources of funding will be received.

Competing interests None declared.

Patient consent for publication Not required.

Ethics approval The proposed study has been approved by the Human Research Ethics Committee of the Central Adelaide Local Health Network, SA Health, Government of South Australia (HREC/18/CALHN/687), and the University of Adelaide (HREC reference no. 33 383).

Provenance and peer review Not commissioned; externally peer reviewed.

Open access This is an open access article distributed in accordance with the Creative Commons Attribution Non Commercial (CC BY-NC 4.0) license, which permits others to distribute, remix, adapt, build upon this work non-commercially, and license their derivative works on different terms, provided the original work is properly cited, appropriate credit is given, any changes made indicated, and the use is non-commercial. See: http://creativecommons.org/licenses/by-nc/4.0/.

\section{ORCID iDs}

Lalit Yadav http://orcid.org/0000-0002-7055-3247

Tiffany K Gill http://orcid.org/0000-0002-2822-2436

Mellick J Chehade https://orcid.org/0000-0001-6931-2393

\section{REFERENCES}

1 Archibald MM, Ambagtsheer R, Beilby J, et al. Perspectives of frailty and frailty screening: protocol for a collaborative knowledge translation approach and qualitative study of stakeholder understandings and experiences. BMC Geriatr 2017;17:87.

2 Falaschi P, Marsh DR, eds. Practical issues in geriatrics: Orthogeriatrics. Switzerland: Springer International Publishing. ISBN 978-3-319-43249-6, 2017
3 Cooper C, Campion G, Melton LJ. Hip fractures in the elderly: a world-wide projection. Osteoporos Int 1992;2:PubMed:1421796:285-9.

4 Gullberg B, Johnell O, Kanis JA. World-Wide projections for hip fracture. Osteoporos Int 1997;7:407-13.

5 Johnell O, Kanis JA. An estimate of the worldwide prevalence, mortality and disability associated with hip fracture. Osteoporos Int 2004; 15:897-902.

6 Cummings SR, Melton LJ. Epidemiology and outcomes of osteoporotic fractures. The Lancet 2002;359:1761-7.

7 Brainsky A, Glick H, Lydick E, et al. The economic cost of hip fractures in community-dwelling older adults: a prospective study. $J$ Am Geriatr Soc 1997;45:281-7.

8 Haentjens P, Autier P, Barette M, et al. The economic cost of hip fractures among elderly women. A one-year, prospective, observational cohort study with matched-pair analysis. Belgian hip fracture Study Group. J Bone Joint Surg Am 2001;83:493-500.

9 Wiktorowicz ME, Goeree R, Papaioannou A, et al. Economic implications of hip fracture: health service use, institutional care and cost in Canada. Osteoporos Int 2001;12:271-8.

10 Sahota O, Morgan N, Moran CG. The direct cost of acute hip fracture care in care home residents in the UK. Osteoporos Int 2012;23:917-20.

11 Australian and New Zealand Hip Fracture Registry (ANZHFR) Steering Group. Australian and New Zealand guideline for hip fracture care: improving outcomes in hip fracture management of adults. Sydney: Australian and New Zealand Hip Fracture Registry Steering Group, 2014.

12 NHS England, South. Safe, compassionate care for frail older people using an integrated care pathway: practical guidance for commissioners, providers and nursing, medical and allied health professional leaders 2014.

13 Hadden KB, Prince LY, Bushmiaer MK, et al. Health literacy and surgery expectations in total hip and knee arthroplasty patients. Patient Educ Couns 2018;101:1823-7.

14 Olsson L-E, Karlsson J, Ekman I. The integrated care pathway reduced the number of hospital days by half: a prospective comparative study of patients with acute hip fracture. J Orthop Surg Res 2006:1:3.

15 Olsson L-E, Hansson E, Ekman I, et al. A cost-effectiveness study of a patient-centred integrated care pathway. J Adv Nurs 2009;65:1626-35.

16 NHS England, Coalition for collaborative care. Personalised care and support planning Handbook: the journey to person-centred care 2016.

17 Harwell MR. Research design in qualitative/quantitative/mixed methods. In: Conrad CF, Serlin RC, eds. The SAGE Handbook for research in education: pursuing ideas as the keystone of exemplary inquiry. In:. Thousand Oaks: SAGE Publications, Inc, 2011: 147-82.

18 DABBS ADEV, Myers BA, Mc Curry KR, et al. User-Centered design and interactive health technologies for patients. CIN: Computers, Informatics, Nursing 2009;27:175-83.

19 Harte R, Glynn L, Rodríguez-Molinero A, et alMoorhead A, ed. A Human-Centered design methodology to enhance the usability, human factors, and user experience of connected health systems: a three-phase methodology. 4. (1)e8. JMIR Human Factors., 2017.

20 Chathoth P, Altinay L, Harrington RJ, et al. Co-production versus co-creation: a process based continuum in the hotel service context. International Journal of Hospitality Management 2013;32:11-20.

21 World Health Organization. Integrated care for older people: guidelines on community-level interventions to manage declines in intrinsic capacity. Geneva: World Health Organization, 2017Licence: CC BY-NC-SA 3.0 IGO.

22 National Clinical Guideline Centre. The management of hip fractures in adults. National clinical guideline centre 2011 (2017 addendum). Available: www.ncgc.ac.uk

23 Kelders SM, Oinas-Kukkonen H, Oörni A, et al. Health behavior change support systems as a research discipline; a viewpoint. Int $J$ Med Inform 2016:96:3-10.

24 Harvey G, Kitson A. PARIHS revisited: from heuristic to integrated framework for the successful implementation of knowledge into practice. Implementation Science 2015;11.

25 Oinas-Kukkonen $\mathrm{H}$. A foundation for the study of behavior change support systems. Pers Ubiquit Comput 2013;17:1223-35.

26 Kitson A, Harvey G, McCormack B. Enabling the implementation of evidence based practice: a conceptual framework. Qual Health Care 1998;7:149-58.

27 Tung H-H, Lu T-M, Chen L-K, et al. Health literacy impact on elderly patients with heart failure in Taiwan. Journal of Clinical Gerontology and Geriatrics 2014:5:72-6. 
28 Shirooka $\mathrm{H}$, Nishiguchi S, Fukutani $\mathrm{N}$, et al. Association between comprehensive health literacy and frailty level in community-dwelling older adults: a cross-sectional study in Japan. Geriatr Gerontol Int 2017:17:804-9.

29 Richtering SS, Morris R, Soh S-E, et al. Examination of an eHealth literacy scale and a health literacy scale in a population with moderate to high cardiovascular risk: Rasch analyses. PLoS One 2017;12:e0175372

30 Thompson MQ, Theou O, Yu S, et al. Frailty prevalence and factors associated with the frailty phenotype and frailty index: findings from the North West Adelaide health study. Australas J Ageing 2018;37:120-6.

31 Riemen AHK, Hutchison JD. The multidisciplinary management of hip fractures in older patients. Orthop Trauma 2016;30:117-22.

32 Gesar B, Baath C, Hedin H, et al. Hip fracture; an interruption that has consequences four months later. A qualitative study. Int $J$ Orthop Trauma Nurs 2017;26:43-8.

33 Gesar B, Hommel A, Hedin H, et al. Older patients' perception of their own capacity to regain pre-fracture function after hip fracture surgery - an explorative qualitative study. Int J Orthop Trauma Nurs 2017;24:50-8.

34 Jensen CM, Smith AC, Overgaard S, et al. "If only had I known": a qualitative study investigating a treatment of patients with a hip fracture with short time stay in hospital. Int J Qual Stud Health Wellbeing 2017;12:1307061.

35 Ellen ME, Shach R, Balicer RD. Helping patients help themselves: supporting the healthcare journey. Patient Educ Couns 2018;101:1708-1711.

36 Sims-Gould J, Stott-Eveneshen S, Fleig L, et al. Patient perspectives on engagement in recovery after hip fracture: a qualitative study. $J$ Aging Res 2017;2017:1-9.
37 Kastner M, Perrier L, Munce SEP, et al. Complex interventions can increase osteoporosis investigations and treatment: a systematic review and meta-analysis. Osteoporos Int 2018;29:5-17.

38 Australian Commission on Safety and Quality in Health Care. Safety and quality improvement guide standard 2: partnering with consumers October 2012. Sydney: ACSQHC, 2012.

39 Whitehead L, Seaton P. The effectiveness of self-management mobile phone and tablet Apps in long-term condition management: a systematic review. J Med Internet Res 2016;18:e97.

40 Kinghan D, Carson P, Flanagan A, et al. (2017) patient education / self management programmes for people with long term conditions (2016/17). Available: https://www.health-ni.gov.uk/sites/default/files/ publications/health/pesmp-Itc-ni-16-17_0.pdf

41 Thompson B. Education and learning for people with ankylosing spondylitis. Newcastle, UK: Newcastle University, 2011.

42 Raybould G, Babatunde O, Evans AL, et al. Expressed information needs of patients with osteoporosis and/or fragility fractures: a systematic review. Arch Osteoporos 2018;13:55.

43 Brookes G, Baker P. What does patient feedback reveal about the NHS? a mixed methods study of comments posted to the NHS choices online service. BMJ Open 2017;7:e013821.

44 Jensen CM, Overgaard S, Wiil UK, et al. Bridging the gap: a user-driven study on new ways to support self-care and empowerment for patients with hip fracture. SAGE Open Medicine 2018;6:205031211879912-3.

45 Jensen CM, Overgaard S, Wiil UK, et al. Can tele-health support self-care and empowerment? A qualitative study of hip fracture patients' experiences with testing an "App". SAGE Open Nurs 2019:5:237796081982575-11.

46 Young HM, Nesbitt TS. Increasing the capacity of primary care through enabling technology. J Gen Intern Med 2017;32:398-403. 